\title{
Erratum: The Herschel Bright Sources (HerBS): sample definition and SCUBA-2 observations
}

\author{
by Tom J. L. C. Bakx ${ }^{\oplus},{ }^{\star}{ }^{\circ}$ S. A. Eales, ${ }^{1}$ M. Negrello, ${ }^{1}$ M. W. L. Smith ${ }^{\odot},{ }^{1}$ E. Valiante, ${ }^{1}$ \\ W. S. Holland, ${ }^{2}$ M. Baes ${ }^{\oplus},{ }^{3}$ N. Bourne ${ }^{\oplus},{ }^{4}$ D. L. Clements ${ }^{\odot 5}$ H. Dannerbauer, ${ }^{6,7,8}$ \\ G. De Zotti, ${ }^{9}$ L. Dunne, ${ }^{1}$ S. Dye ${ }^{\odot},{ }^{10}$ C. Furlanetto ${ }^{\odot},{ }^{10,11}$ R. J. Ivison ${ }^{\oplus},{ }^{4,12}$ S. Maddox,${ }^{1}$ \\ L. Marchetti ${ }^{\oplus},{ }^{13}$ M. J. Michałowski ${ }^{\oplus},{ }^{4,14}$ A. Omont, ${ }^{15,16}$ I. Oteo, ${ }^{4,12}$ J. L. Wardlow, ${ }^{17}$ \\ P. van der Werf $^{18}$ and C. Yang ${ }^{\oplus 15,16,19,20,21}$ \\ ${ }^{1}$ School of Physics and Astronomy, Cardiff University, The Parade, Cardiff, CF24 3AA, UK \\ ${ }^{2}$ UK Astronomy Technology Centre, Royal Observatory, Blackford Hill, Edinburgh EH9 3HJ, UK \\ ${ }^{3}$ Sterrenkundig Observatorium, Universiteit Gent, Krijgslaan 281 S9, B-9000 Gent, Belgium \\ ${ }^{4}$ Institute for Astronomy, University of Edinburgh, Royal Observatory, Edinburgh EH9 3HJ, UK \\ ${ }^{5}$ Astrophysics Group, Imperial College, Blackett Laboratory, Prince Consort Road, London SW7 2AZ, UK \\ ${ }^{6}$ Institut für Astrophysik, Universität Wien, Türkenschanzstrabe 17, A-1180 Wien, Austria \\ ${ }^{7}$ Instituto de Astrofísica de Canarias (IAC), E-38205 La Laguna, Tenerife, Spain \\ ${ }^{8}$ Dpto. Astrofísica, Universidad de La Laguna, E-38206 La Laguna, Tenerife, Spain \\ ${ }^{9}$ INAF - Osservatorio Astronomico di Padova, Vicolo dell'Osservatorio 5, I-35122 Padova, Italy \\ ${ }^{10}$ School of Physics and Astronomy, University of Nottingham, University Park, Nottingham NG7 2RD, UK \\ ${ }^{11}$ CAPES Foundation, Ministry of Education of Brazil, 70040-020 Brasília, DF, Brazil \\ ${ }^{12}$ European Southern Observatory, Karl-Schwarzschild-Strasse 2, D-85748 Garching bei München, Germany \\ ${ }^{13}$ Department of Physical Science, The Open University, Milton Keynes MK7 6AA, UK \\ ${ }^{14}$ Astronomical Observatory Institute, Faculty of Physics, Adam Mickiewicz University, ul. Stoneczna 36, PL-60-286 Poznań, Poland \\ ${ }^{15}$ UPMC Univ. Paris 06, UMR7095, Institut d'Astrophysique de Paris, F-75014 Paris, France \\ ${ }^{16}$ CNRS, UMR7095, Institut d'Astrophysique de Paris, F-75014 Paris, France \\ ${ }^{17}$ Centre for Extragalactic Astronomy, Department of Physics, Durham University, South Road, Durham, DH1 3LE, UK \\ ${ }^{18}$ Leiden Observatory, Leiden University, PO Box 9513, NL-2300 RA Leiden, the Netherlands \\ ${ }^{19}$ Purple Mountain Observatory/Key Lab of Radio Astronomy, Chinese Academy of Sciences, Nanjing 210008, China \\ ${ }^{20}$ Institut d'Astrophysique Spatiale, CNRS, Univ. Paris-Sud, Université Paris-Saclay, Bât. 121, F-91405 Orsay Cedex, France \\ ${ }^{21}$ Graduate University of the Chinese Academy of Sciences, 19A Yuquan Road, Shijingshan District, Beijing 10049, China
}

Key words: errata, addenda-gravitational lensing: strong-galaxies: high-redshift-submillimetre: galaxies.

In the original paper, Bakx et al. (2018) extracted the SCUBA-2 fluxes by a method that has later been shown to result in fluxes that are too high by around 50 to 75 per cent. In this erratum, we present fluxes extracted with the proven method as shown in Ivison et al. (2016), Holland et al. (2017) and Duivenvoorden et al. (2018), in an effort to present the most complete and correct catalogue of fluxes and photometric redshifts, shown in Table A1. Adding to the list of sources with SCUBA-2 fluxes, we include thirteen sources from the 2015 SCUBA-2 project M15AI96 (PI: S. Eales). Similarly, we drop two sources that recently have been shown to be blazar contaminants, namely HerBS-16 and HerBS-112.

All aspects of the data reduction are the same, except the method we use to reduce the raw SCUBA-2 data into calibrated postprocessed data. In our new data reduction method, we use the 'zero-mask' method (Holland et al. 2017), which uses the Dynamic Iterative Map Maker within the SMURF package (Chapin et al. 2013). This procedure assumes that the only astronomical signal is within a 60 arcsec diameter region centered on our target. We

\footnotetext{
^E-mail: bakx@a.phys.nagoya-u.ac.jp
}

then apply a matched filter, where we convolve the image with the beam-size $(\sim 13 \operatorname{arcsec})$. The pixel size of the final maps is one by one arcsec.

We use the same data reduction technique on the flux calibrators to get an accurate flux conversion factor (FCF). The FCF for each source was derived by calibrator observations taken in the same night, and are linearly interpolated between multiple calibrator sources, depending on the time of the observation of the source. These FCFs range between 575 and $760 \mathrm{mJy} \mathrm{pW}^{-1}$ beam $^{-1}$, in range with the values seen in Duivenvoorden et al. (2018). The calibration uncertainty is expected to be accurate to around 5 per cent (Dempsey et al. 2013).

We extract the fluxes by the brightest pixel within a $20 \operatorname{arcsec}$ radius around the central position. We visually inspect the sources to ensure the correct source is extracted, which is true in all cases. We derive the uncertainty in the flux to be the standard deviation of the off-source pixels ( $>25$ arcsec from the target position) within a 3' box centered on the target position, where we expect a uniform observation depth.

The difference in the $850 \mu \mathrm{m}$ flux density did not significantly change the fitted parameters of the modified black-body fit, and 


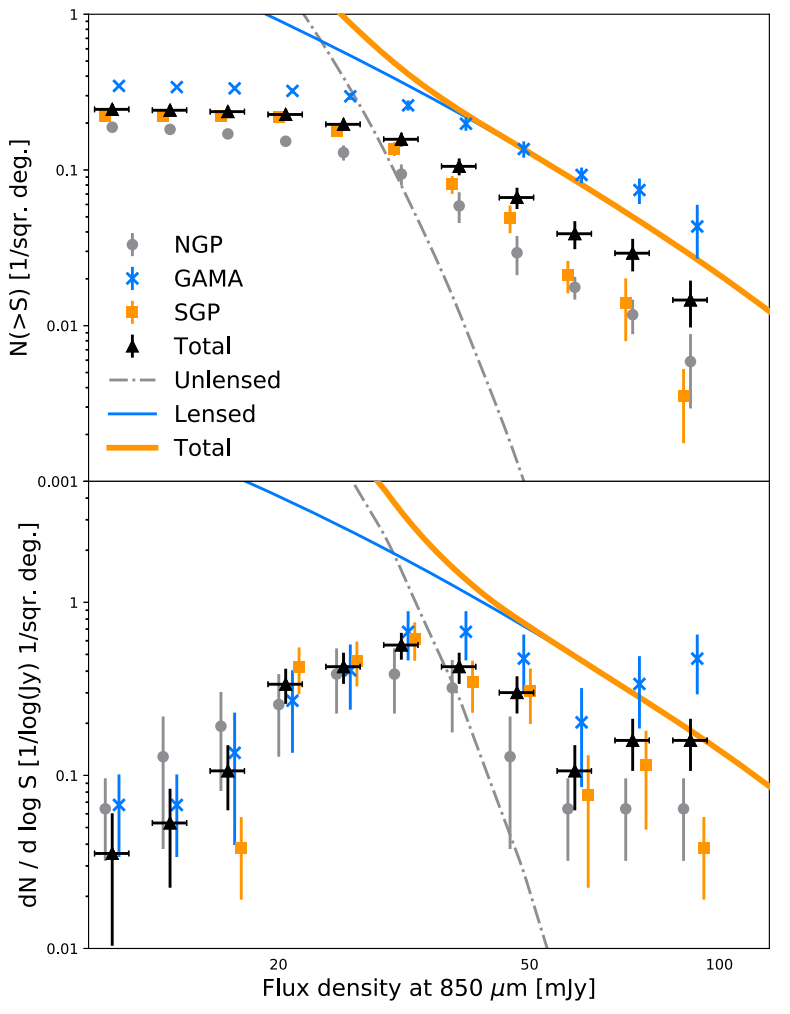

Figure 1. Revised version of fig. 10 from Bakx et al. (2018). The top panel shows the cumulative number counts and the bottom panel shows the differential number counts of our HerBS sample, compared to the predictions of the model of Cai et al. (2013) for unlensed (dashed grey line) and lensed (solid blue line) galaxies. the new photometric redshifts are not systemically different to the previous photometric redshifts (15 percent), although improved photometry is crucial for our ongoing redshift searches. The only significant change from the previous paper is to the estimated fraction of lenses. We show an updated version of Figure 10 from Bakx et al. (2018) in Fig. 1. This figure shows the cumulative (top panel) and differential (bottom panel) for the $850 \mu \mathrm{m}$ fluxes of the HerBS sources. We note that the model by Cai et al. (2013) slightly over-predicts the source counts seen for the HerBS sources. We also note higher source counts for HerBS sources in the GAMA fields compared to the Northern and Southern Galactic Pole (NGP and SGP fields).

\section{REFERENCES}

Bakx T. J. L. C. et al., 2018, MNRAS, 473, 1751

Cai Z.-Y. et al., 2013, ApJ, 768, 21

kChapin E. L. et al., 2013, MNRAS, 430, 2545

Dempsey J. T. et al., 2013, MNRAS, 430, 2534

Duivenvoorden S. et al., 2018, MNRAS, 477, 1099

Holland W. S. et al., 2017, MNRAS, 470, 3606

Ivison R. J. et al., 2016, ApJ, 832, 78

Negrello M. et al., 2017, MNRAS, 465, 3558

\section{APPENDIX A: HERBS CATALOGUE AND BLAZARS}




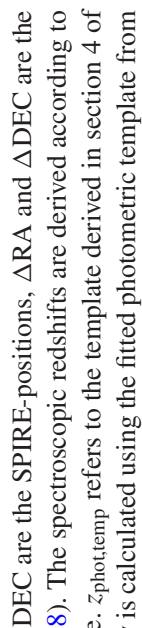

需这

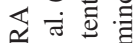

过

证

它宕

क m o

동

讨

호 $\cong$

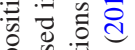

密

$\pm$

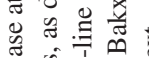

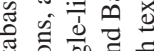

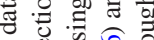

곤 원은

政

의

क च :

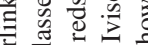

证

일 항

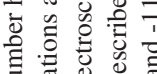

要盛

की

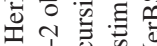

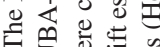

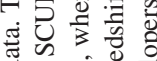

क人

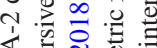

击

论 宛

请

I

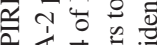

ज㐫㐫ฐ

는

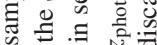

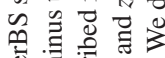

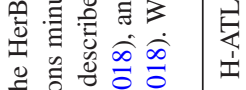

당 웡

- : ठ न

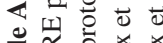

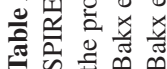

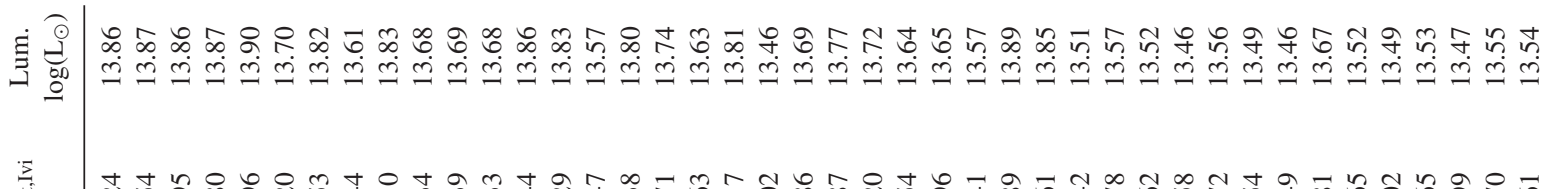

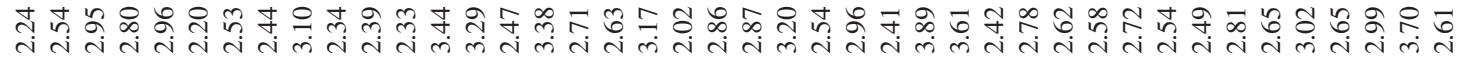

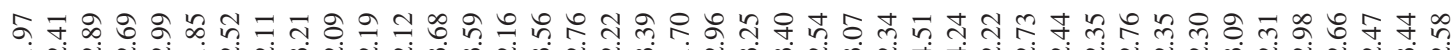

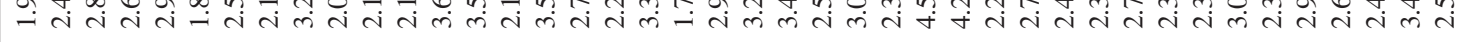

幾

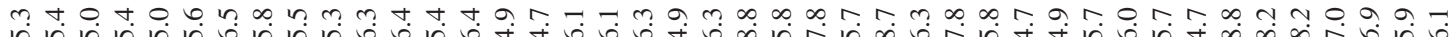
在H H H H H H H H H H H H H H H H H H H H H H H H H H H H H H H H H H H H H H H H H H m

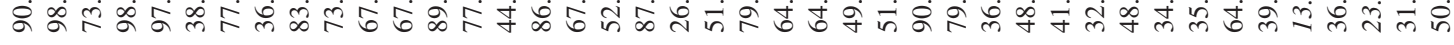

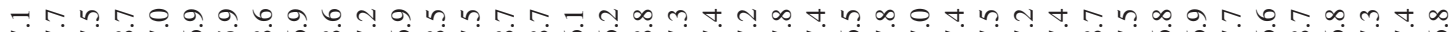

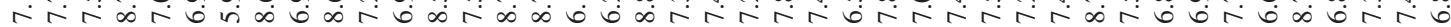
$H+H+H+H+H+H+H+H+H+H+H+H+H+H+H+H+H+H+H+H+H+H+H+H+H+H+H+H$ m

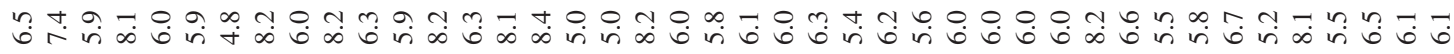
紋 H H H H H H H H H H H H H H H H H H H H H H H H H H H H H H H H H H H H H H H H H H

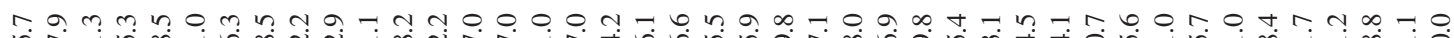

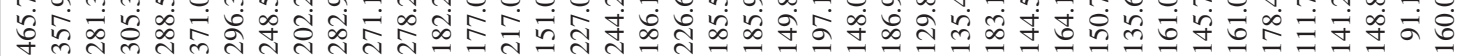

舟 在H H H H H H H H H H H H H H H H H H H H H H H H H H H H H H H H H H H H นึ่ छี a 웅

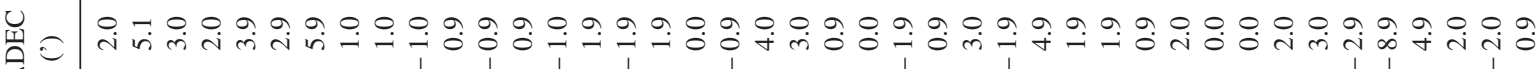
ঝ

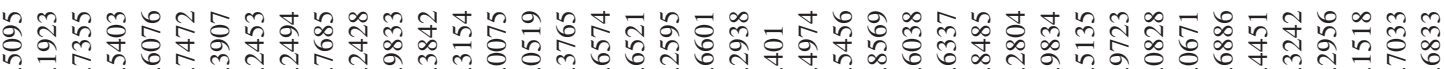

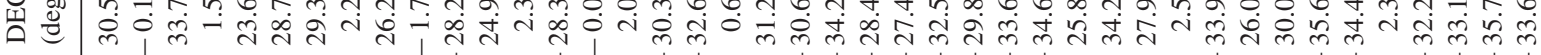

赵设

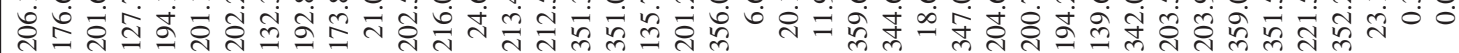

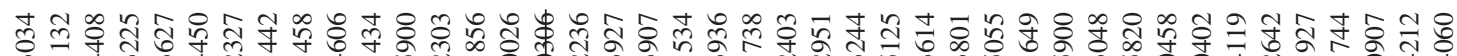

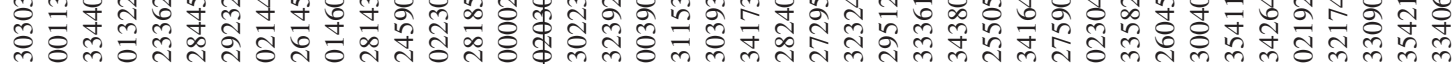

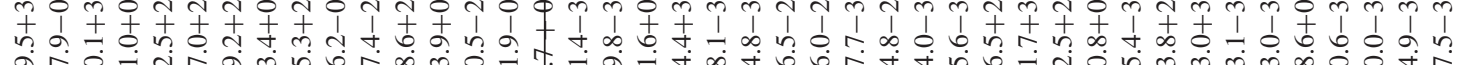

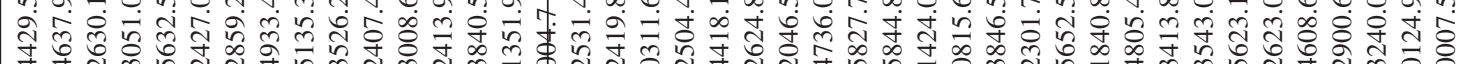

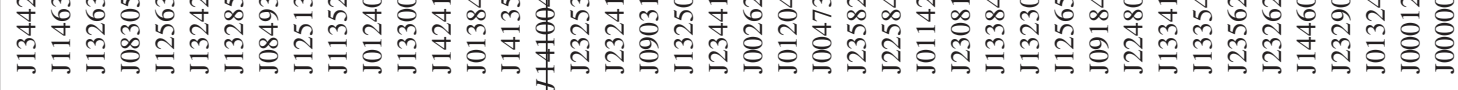




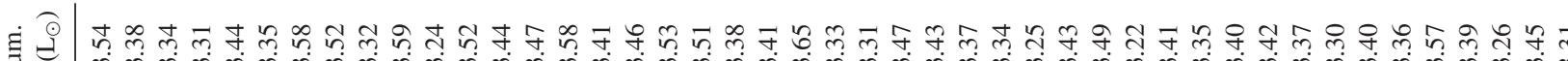

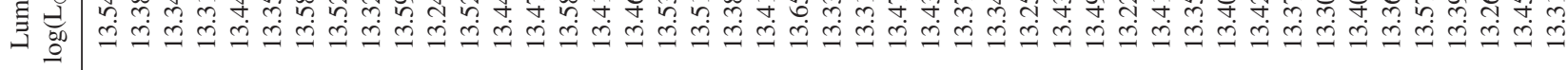

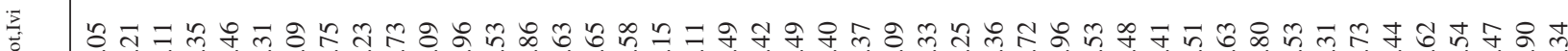
बंतु

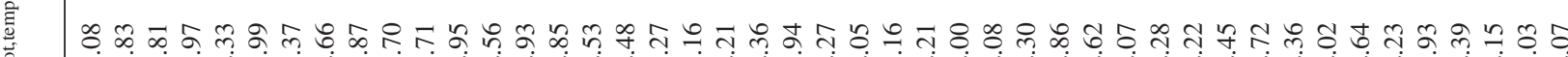
㠃

产

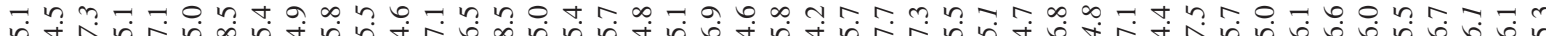

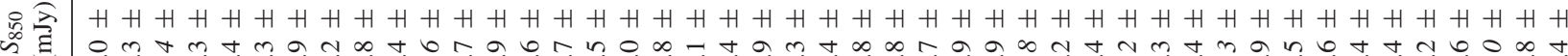

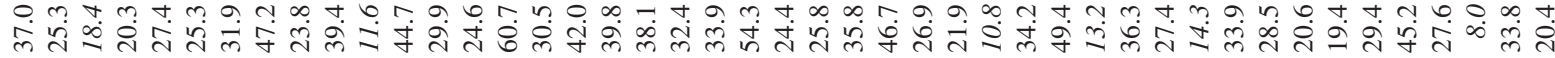

m $H+H+H+H+H+H+H+H+H+H+H+H+H+H+H+H+H+H+H+H+H+H+H+H+H+H$ 获卞

그

在H H H H H H H H H H H H H H H H H H H H H H H H H H H H H H H H H H H H H H H H H H H + H

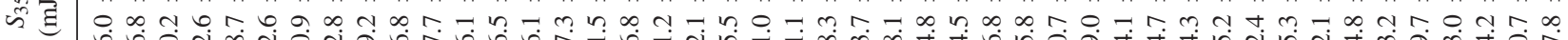
向

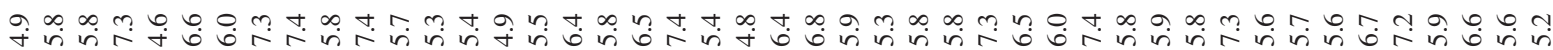
续H H H H H H H H H H H H H H H H H H H H H H H H H H H H H H H H H H H H

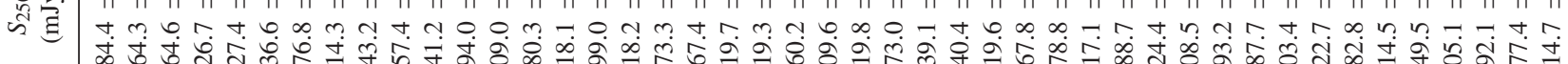

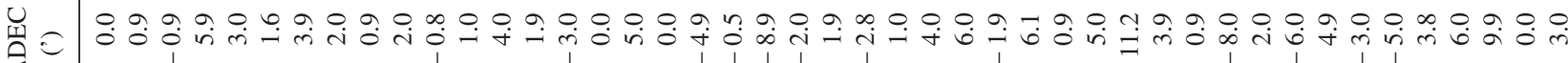

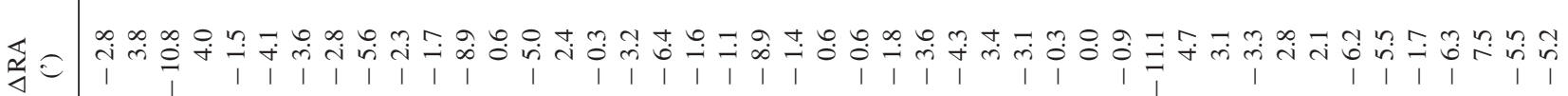

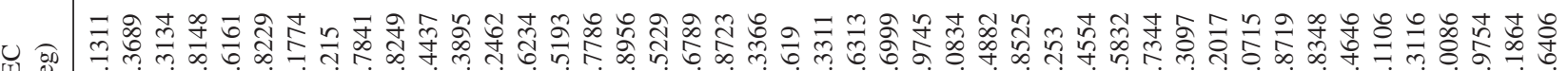
荘

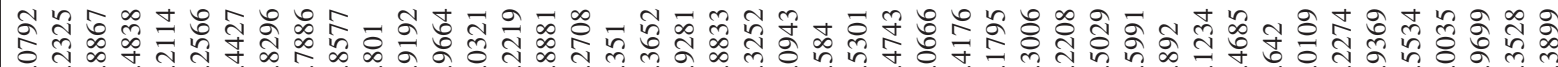
穴穴

두유

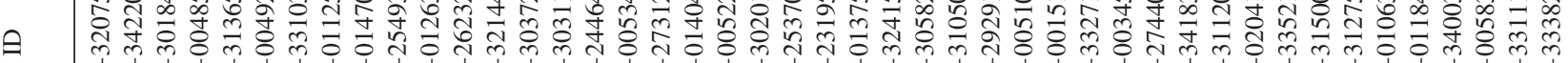

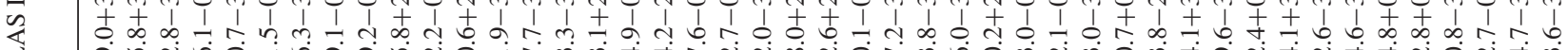

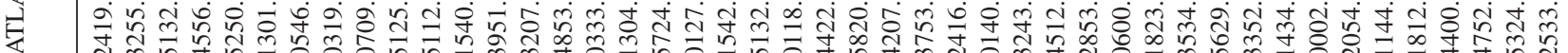
I 


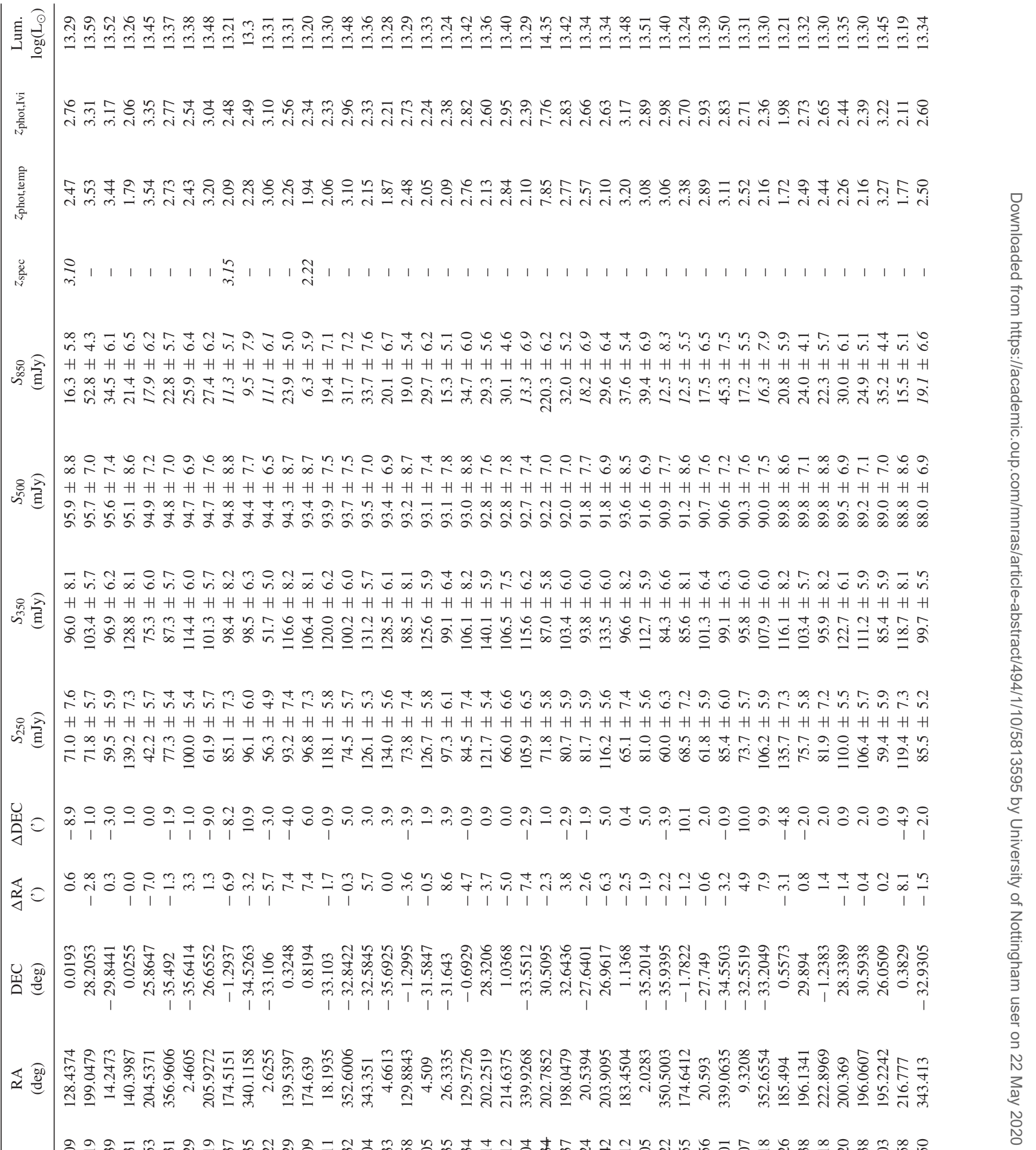

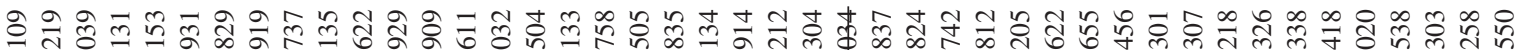

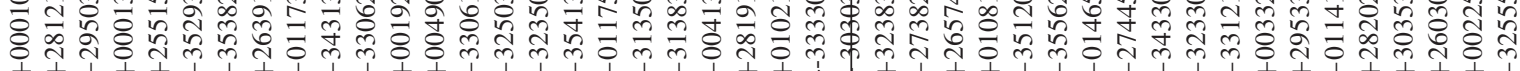

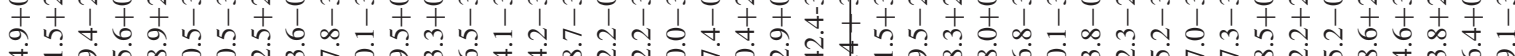

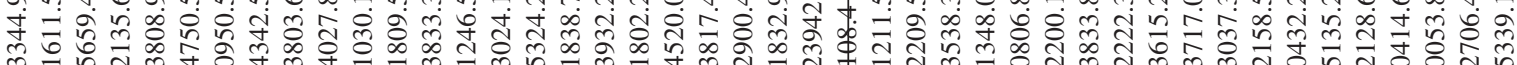

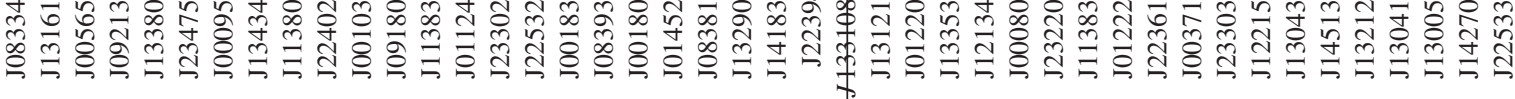


घं

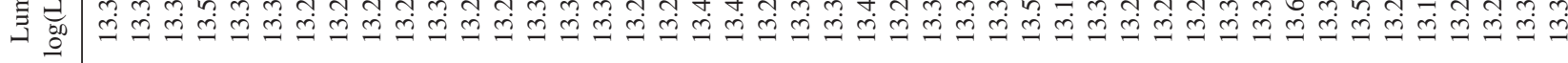

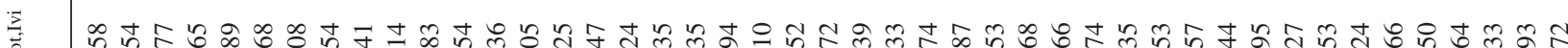

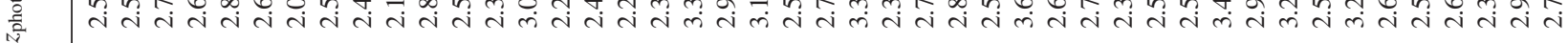

产 तथ

产

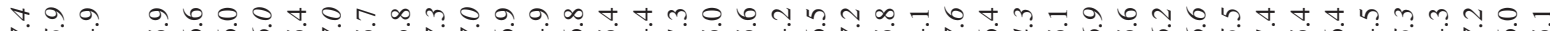
质H H H H H H H H H H H H H H H H H H H H H H H H H H H H H H H H H H H H H H H H H H

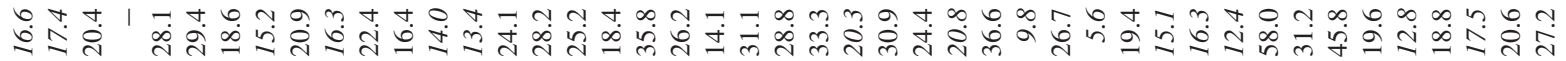

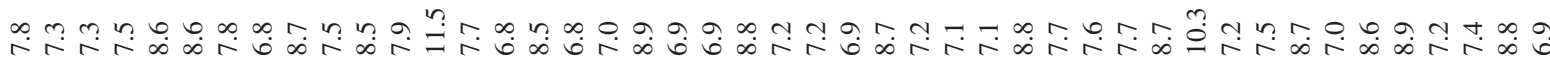
$H+H+H+H+H+H+H+H+H+H+H+H+H+H+H+H+H+H+H+H+H+H+H+H+H+H+H$

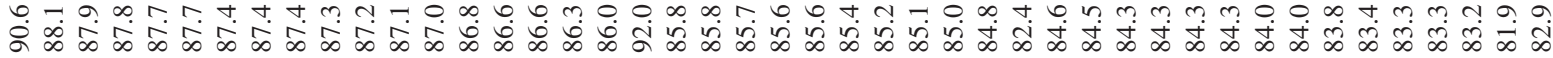

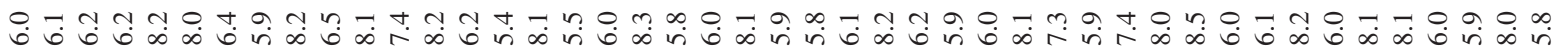
合H H H H H H H H H H H H H H H H H H H H H H H H H H H H H H H H H H H H H H H H H H H H H

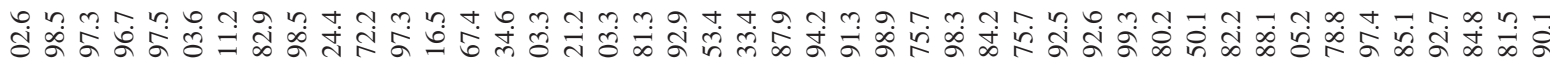

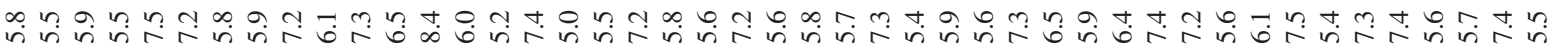
$H+H+H+H+H+H+H+H+H+H+H+H+H+H+H+H+H+H+H+H+H+H+H+H+H+H+H+H+H+H+H+H$

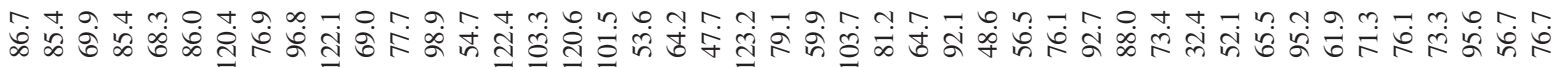

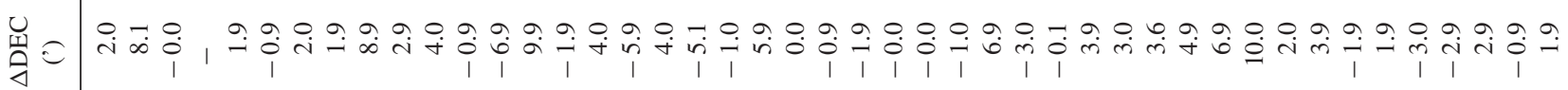
『)

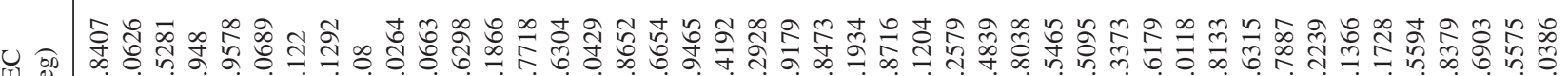
ग्र

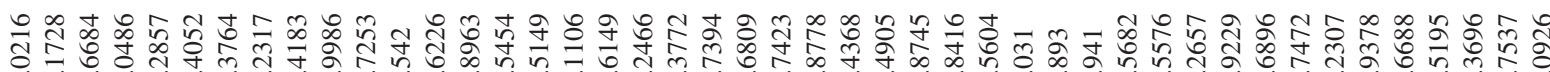

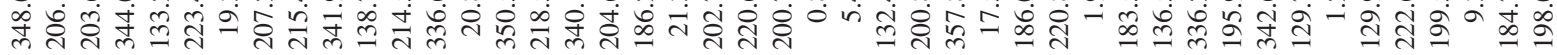

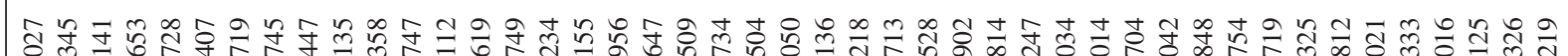
a 술

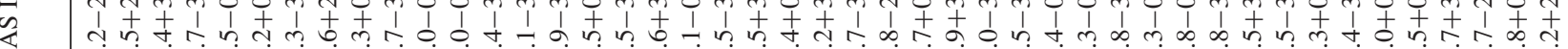

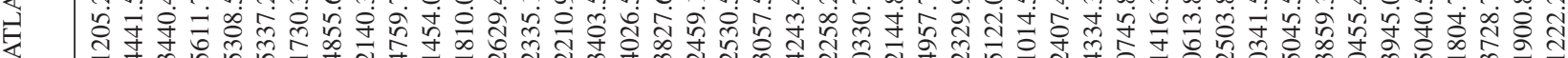

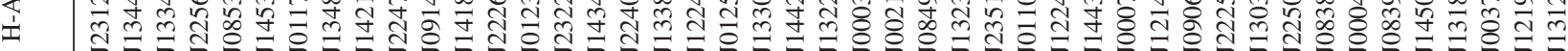




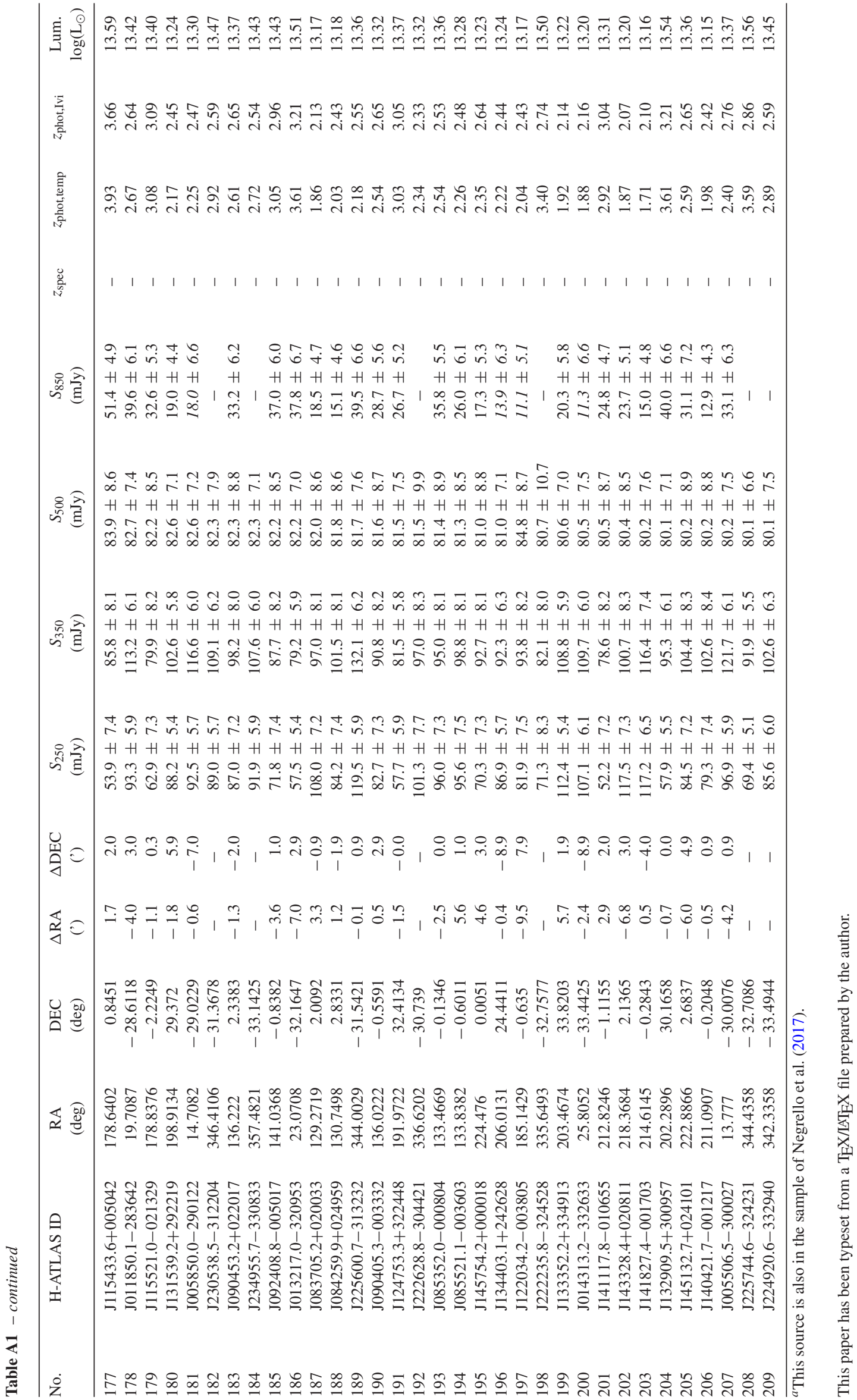

